\title{
Cardiovascular Manifestation
}

National Cancer Institute

\section{Source}

National Cancer Institute. Cardiovascular Manifestation. NCI Thesaurus. Code C27231.

Patient complaints (symptoms), physical/laboratory findings(signs) related to the cardiovascular system. 